\title{
Aging Agents: Cross Generational Analysis of Behavior and Need Satisfaction Among Players of Tom Clancy's The Division 2
}

\author{
Ahmad Azadvar ${ }^{1,2} \cdot$ Ebba Dalqvist ${ }^{1}$ \\ Published online: 9 March 2020 \\ (c) The Author(s) 2020
}

\begin{abstract}
This research investigated the effect of age on players of an online multiplayer shooter. Through combining the data from two large scale surveys, we collected information regarding age, gaming habits, game rating and psychological need satisfaction for 8120 players of Tom Clancy's The Division. Behavioral data extracted from the game's tracking engine was then cross-referenced for different age groups to indicate motivational, behavioral and habitual characteristics of each age group. To find the importance of measured factors we employed a rank-based model for comparing independent sample means for intergenerational analysis (Kendall's tau for non-parametric correlations) as well as multiple Machine Learning algorithms. Results found that different measures of playtime vary significantly among generations. Baby Boomers showed significantly higher playtime, days played and group playtime. Intergenerational comparison of perceived need satisfaction also found that older gamers feel more agentic, present in the narrative, closer to non-playable characters but less competent at the game. Percentage of group playtime also showed a decrease in older generations. Future research may expand cross generational analysis to other game types and include more granular behavioral measures.
\end{abstract}

Keywords Behavior modeling · Affective computing · Social play ·

Intergenerational $\cdot$ Older adults $\cdot$ Video games

Ahmad Azadvar

ahmad.azadvar@mau.se

Ebba Dalqvist

Ebba.dalqvist@massive.se

1 Ubisoft Entertainment Sweden, Drottninggatan 34, 21141 Malmö, Sweden

2 Department of Computer Science and Media Technology, Faculty of Technology and Society,

Malmö University, Nordenskiöldsgatan 1, 21119 Malmö, Sweden 


\section{Introduction}

Academic research on video games as a cultural phenomenon dates to the early 2000s (Mäyrä 2008) although commercial games have been on the market since 1971 (Kent 2010). However, perhaps the economic significance of the games industry was made apparent in 2018 when video game revenues surpassed projected box office for the film industry in the United States (Entertainment Software Association 2018).

Implications of this considerable contribution of video games in the day-today leisure activities of the consumer have been largely reduced to dependence (Fisher 1994; Skoric et al. 2009; Han et al. 2011) and relationship to violence (Sherry 2001; Carnagey et al. 2007; Dill and Dill 1998). Yet, there has been additional contributions such as the cognitive effects (Green and Bavelier 2003) and learning (Prensky 2003); while epistemology, ethics (Jenson and de Castell 2018) or politics (Hong 2015) have rarely been addressed.

One implication is the diversity of audiences that this medium attracts and the potential differences in motivations (Ryan et al. 2006) and behaviors they perform within the game (Canossa et al. 2015) as well as how they perceive the quality of its content (Huang et al. 2017). However, academics have seldom looked at the combined effects of motivations and player behavior (Azadvar and Canossa 2018), or game evaluation and age (Salmon et al. 2017).

This study aims to examine the age and intergenerational relationships as vectors of diversity in an online multiplayer video game, Tom Clancy's The Division 2 (TD2) (Ubisoft Entertainment Sweden 2019) by looking at both motivations and player behavior data.

\section{Methods}

\subsection{Background}

If we assume the definitions of the PEW Research Center (2010), a popular multiplayer online shooter such as TD2 represents players of at least five distinct generations, which makes it a suitable environment for the study of intergenerational gaming (Schotter and Sopher 2003).

We focused on more frequently available data from four generations of Baby Boomers, Generation X, Millennials and Generation Z players (for a precise definition see the Sect. "2.2"). Previous studies of intergenerational gaming have largely focused on the intergenerational interaction that games enable and its beneficial impacts, such as increased understanding of other generations and reduced social anxiousness (De la Hera et al. 2017). Reviewing the factors and benefits of designing intergenerational games, De la Hera et al. (2017) argue that it is necessary to take a closer look into the effects of age and gender on benefits of playing digital games. 
Furthermore, while some research has focused specifically on a single generation in order to identify factors such as gaming habits and reasons to play (e.g. De Schutter 2011; Pearce 2008; Marston and Graner-Ray 2016), the current study will expand on this topic by investigating similarities and differences between generations.

In this study, two large scale surveys were distributed among players of $T D 2$, comprising of demographic data such as age, a 10-point scale rating of the game, the Ubisoft Perceived Experience Questionnaire (UPEQ) (Azadvar and Canossa 2018), and an extracted list of behavioral measures from participants' gameplay.

\subsection{Aims and Objectives}

The goal of the study is to explore the relationships between age groups and players' attributes such as game ratings, evaluation of need satisfaction (via UPEQ) and ingame behaviors of the players.

By including a large age span and making comparisons between different generational categories, the current study aims to expand on previous research and contribute with new insights to the field.

\subsection{Participants}

A survey was deployed among the players of TD2, targeting one hundred thousand active players. Active players are defined as players who have at least one play session lasting more than $1 \mathrm{~h}$ in the past 2 weeks. No further filtering of the participants was administered to ensure a representative sampling of all players.

The generational categories used are adopted from standards set by PEW Center (2010), and are as follows:

- Generation Z, 22 years old and younger, $(\mathrm{N}=1664$, Mean $=19.51, \mathrm{SD}=1.859)$

- Millennials, between 23 and 38 years old, $(\mathrm{N}=4297, \mathrm{Mean}=30.12, \mathrm{SD}=4.418)$

- Generation $X$, between 39 to 54 years old, $(N=1856$, Mean $=44.67, S D=4.17)$

- Baby Boomers, between 55 to 75 years old, $(\mathrm{N}=303$, Mean =60.33, $\mathrm{SD}=5.019)$.

\subsection{Measures}

The survey used for this study required players to rate their experience with the game based on a 10-point Likert scale, and to answer 21 questions rated on a 5-point Likert scale questionnaire known as UPEQ (Azadvar and Canossa 2018). This questionnaire aims to measure players' perceived psychological need satisfaction as described by the self-determination theory (Deci and Ryan 2012).

The UPEQ is a 30-item survey (“Appendix 1"), comprising of 3-items per subfactor and four main factors. Three of these are from the self-determination theory, namely Autonomy, Competence and Relatedness, while the fourth, Presence, aims to 
measure the extent to which the respondent feels present in the storyline (Narrative presence), as well as an emotional and physical sense of presence.

Presence, as the illusory state of perceptual non-mediation (Ivory and Kalyanaraman 2007) is an addition to measures of need satisfaction in UPEQ. Autonomy, referring to the sense of volition and freedom to choose from meaningful options that are equally appealing to the player, has two sub factors of Playstyle (freedom and having options) and Agency (choice impact).

Competence is the feeling of being effective while growing, and it includes not only providing and insinuating opportunities for the player to demonstrate their acquired skills, but also the feedback they receive upon completion of a task. The subfactors of Competence are Mastery (performance and feedback) and Growth (progression and increased complexity).

Finally, Relatedness gauges the feeling of belonging and being recognized by others, including non-playable characters (NPCs). Relatedness has three sub factors of Closeness (being together), Interdependence (role impact, social status) and NPC Closeness (being recognized by non-playable-characters) (Azadvar and Canossa 2018).

\subsection{Procedure}

Two surveys were deployed to gamers of TD2, in May of 2019. Questions included: player demographics, namely age, gender identity, self-reported measures of gaming time per week and money spent on video games per month, platform of play, and favorite games (an open-ended question).

These surveys were created in the Survey Monkey platform and distributed through emailing a link to a random sample of participants. No personal or identifying information was stored in the survey servers. Data collection lasted 2 weeks for both surveys and extraction of behavioral data for all players was conducted at the same time and through Ubisoft's internal server with anonymization. Respondents were divided into four distinct generations as defined in the Sect. "2.2", and relevant behavioral measures, including playtime intensity and frequency, social play (group playtime) and progression in the game, were added to the database.

\subsubsection{Data Collection and Analysis}

Once the survey data was collected, researchers used an anonymized unique player identification number to retrieve player behavioral data from the game's tracking engine.

The behavioral measures included in this study were "daysplayed" (number of days in which the player has at least one play-session), "daysingroup" (number of days in which the player has at least one play-session involving other players), "playtime hrs" (total amount of time spent by the player in the game by hours), "playtimegroup hrs" (total amount of time spent by the player in the game involving other 
players by hours), "prelvl30 playtime hrs" (total amount of time spent by the player in the game before reaching the narrative end, by hours), "prelvl30 playtimegroup hrs" (total amount of time spent by the player in the game involving other players before reaching the narrative end, by hours), "max gearscore" (an in-game measure of progression tied to the quality of the character's gear), and "max level" (an ingame measure of progression tied to completion of activities which grant experience points).

The data collected from both survey's respondents was then cleaned and categorised by the age data, which resulted in a total of 8120 responses divided into four generations: Baby Boomers, Generation X, Millennials, and Generation Z.

In the dataset, coding was conducted via different generational labels which facilitated the next step to examine whether there were significant differences in average values of different generations.

Data analysis included The Kruskal-Wallis H test (Kruskal and Wallis 1952), a rank-based nonparametric test that does not assume normality of the samples, was employed to determine differences in average values of the tested variables. Then a pairwise comparison between each generation was done to show which two generations showed significant differences.

To further assess the relationship between the variables at hand, a correlational table, reporting the non-parametric measure Kendall's Tau (Kendall 1938), was used to identify significant correlations between age and generation index and other measured variables.

Finally, machine learning methods were employed in order to examine the importance of collected variables in predicting age and generation index of our respondents: a multilayer perceptron neural network (Rumelhart et al. 1986) and a support vector machine algorithm (Cortes and Vapnik 1995), combined with our previous ranked based linear model, into a supervised learning ensemble model (Dietterich 2000).

\subsection{Ethics}

Emailing participants, extraction of behavioral data and processing of self-reported information was conducted through a secondary data processing platform which uses a unique identifier number and a double blinded system to connect individual's emails to their behavioral profile. Recording of these data is conducted separately and is in accordance with GDPR regulations (Zerlang 2017).

\section{Results}

The UPEQ questionnaire showed reliability (between-item reliability $\alpha=0.930$, main factors $\alpha=0.930$ and between subfactors $\alpha=0.915$ ) and all its main and subfactors significantly correlated with the rating of the game. Although rating itself 
showed a negative correlation $(\tau=-0.033)$ with 'playtime hrs' and 'daysplayed' $(\tau=-0.043)$, it is assumed that higher rating as well as higher scores on factors and subfactors of UPEQ indicate a higher satisfaction with the game in general.

Over 200 pairs of Kruskal-Wallis H tests were conducted; therefore, we only report the differences that are shown to reject the null hypothesis- 0.05 significance level, the distributions of the testing variable's two samples (generations in this case) are the same.

Among multiple factors of motivation, there were significant differences regarding Playstyle between Millennials $(\mathrm{M}=3.82, \mathrm{SD}=1.03)$ and both Generation $\mathrm{Z}(\mathrm{M}=3.91, \mathrm{SD}=1.02)$ and Baby Boomers $(\mathrm{M}=4.01, \mathrm{SD}=0.91)$.

Agency displayed the greatest number of significant pairwise comparisons across the generations. Although the difference between Generation $\mathrm{Z}(\mathrm{M}=3.19$, $\mathrm{SD}=1.13)$ and Millennials $(\mathrm{M}=3.14, \mathrm{SD}=1.0)$ did not reject the null hypothesis, all other comparisons, including with Baby Boomers $(\mathrm{M}=3.63, \mathrm{SD}=0.98)$ and Generation $\mathrm{X}(\mathrm{M}=3.35, \mathrm{SD}=1.01)$, were significant.

For Growth, only Baby Boomers $(\mathrm{M}=4.33, \mathrm{SD}=0.86)$ showed a significant difference across the four generations. While Generation $X$ showed $(M=4.18$, $\mathrm{SD}=0.85)$ similar means to Millennials $(\mathrm{M}=4.12, \mathrm{SD}=0.91)$ and Generation $\mathrm{Z}$, $(\mathrm{M}=4.17, \mathrm{SD}=0.91)$.

Mastery saw significant differences between Generation $X \quad(M=3.97$, $\mathrm{SD}=0.8)$, and both Millennials $(\mathrm{M}=4.03, \mathrm{SD}=0.85)$ and Generation $\mathrm{Z}$ $(\mathrm{M}=4.08, \mathrm{SD}=0.84)$.

Closeness displayed one significant difference, namely between Baby Boomers $(\mathrm{M}=3.62, \mathrm{SD}=1.2)$ and Generation $\mathrm{X}(\mathrm{M}=3.48, \mathrm{SD}=1.17)$.

The distribution of ranks in Interdependence was the same across the different generations. While Closeness to non-playable characters (NPC Closeness) was not significantly different between Baby Boomers $(\mathrm{M}=3.15, \mathrm{SD}=1.11)$, Generation $\mathrm{X}$ $(\mathrm{M}=3.07, \mathrm{SD}=1.07)$ or between Millennials $(\mathrm{M}=2.83, \mathrm{SD}=1.17)$ and Generation $\mathrm{Z}(\mathrm{M}=2.85, \mathrm{SD}=1.22)$, it was significant for all other comparisons.

For measures of Presence, only narrative presence showed a significant difference in three degrees. Baby Boomers $(M=3.76, S D=0.85)$ had significant mean differences with both Millennials $(\mathrm{M}=3.55, \mathrm{SD}=1.00)$ and Generation $\mathrm{Z}$ $(\mathrm{M}=3.54, \mathrm{SD}=0.99)$. The third significant mean difference was between Generation $\mathrm{X}(\mathrm{M}=3.65, \mathrm{SD}=0.9)$ and Millennials $(\mathrm{M}=3.55, \mathrm{SD}=1.0)$.

A variety of the Behavioral measures were more prominent, such as 'daysplayed', 'playtime hrs', prelvl30 playtime', rejecting the null hypothesis for all six pairwise comparisons and only Baby Boomers and Generation X being the same for 'daysingroup', 'playtime group hrs', 'prelvl30 playtimegroup' and 'max gearscore'. 'Max level' was the only variable that showed significant differences between Generation $\mathrm{Z}$ and other groups as well as between Millennials and Generation X. Means and standard deviations for these measures are presented in Table 1.

Based on the self-reported measures, Rating of the game only differed between Baby Boomers $(\mathrm{M}=8.16, \mathrm{SD}=1.81)$ and Millennials $(\mathrm{M}=7.91, \mathrm{SD}=1.79)$; Players self-reported the number of hours per week which were spent on playing video 


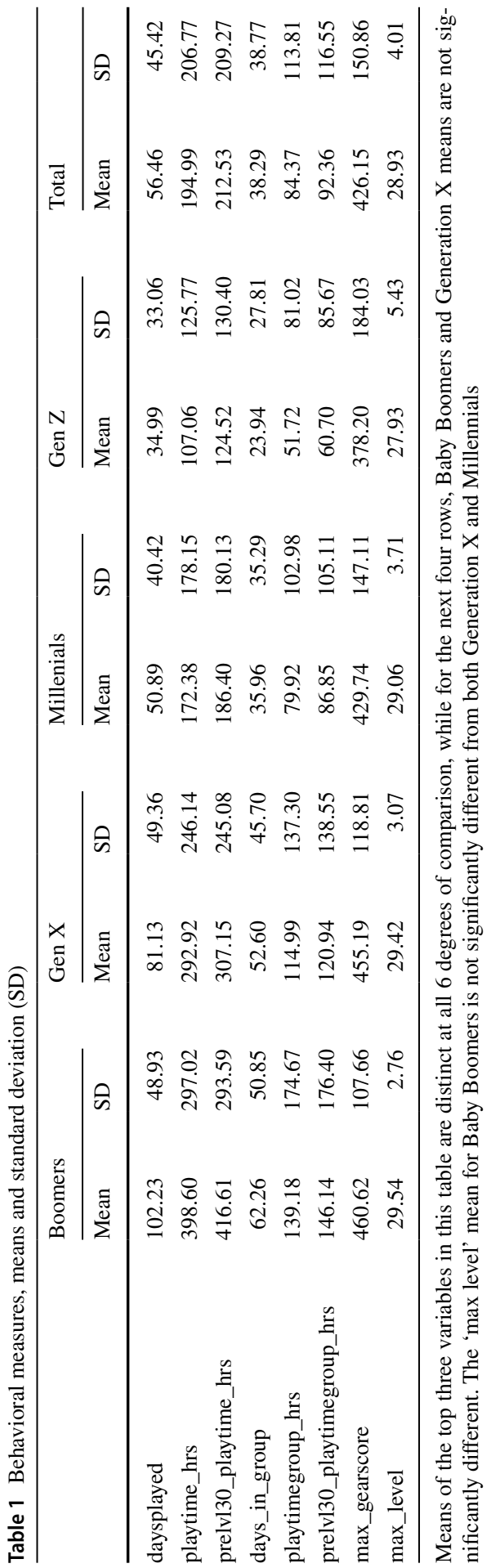


Table 2 Kendall's tau (correlation coefficient)

\begin{tabular}{lcc}
\hline Variables & Age & Generation \\
\hline daysplayed & $.314^{* *}$ & $-.324^{* *}$ \\
daysingroup & $.205^{* *}$ & $-.209^{* *}$ \\
playtime_hrs & $.313^{* *}$ & $-.324^{* *}$ \\
playtimegroup_hrs & $.149^{* *}$ & $-.153^{* *}$ \\
prelvl30_playtime_hrs & $.317^{* *}$ & $-.326^{* *}$ \\
prelvl30_playtimegroup_hrs & $.138^{* *}$ & $-.139^{* *}$ \\
max_gearscore & $.171^{* *}$ & $-.177^{* *}$ \\
max_level & $.115^{* *}$ & $-.121^{* *}$ \\
Self-reported hrs/week & $-.079^{* *}$ & $.084 * *$ \\
Self-reported \$/month & $-.045^{* *}$ & $.041^{* *}$ \\
Rating & 0.01 & -0.01 \\
Playstyle & -0.01 & 0.01 \\
Agency & $.053^{* *}$ & $-.055^{* *}$ \\
Growth & 0.01 & -0.01 \\
Mastery & $-.049^{* * *}$ & $.050^{* *}$ \\
Closeness & -0.01 & 0.01 \\
Interdependence & 0.00 & 0.00 \\
NPCCloseness & $.060^{* *}$ & $-.057^{* *}$ \\
Narrative & $.035^{* *}$ & $-.033^{* *}$ \\
Emotional & 0.01 & -0.01 \\
Physical & 0.00 & 0.00 \\
Autonomy & $.031^{* *}$ & $-.034^{* *}$ \\
Competence & $-.022^{* *}$ & $.023 * *$ \\
Relatedness & 0.00 & 0.00 \\
Age & 1.00 & $-.802^{* *}$ \\
Generation & $-.802^{* *}$ & \\
\hline & & \\
\hline
\end{tabular}

**Correlation is significant at the 0.01 level (2-tailed)

games, and the sole difference identified for this measure was for Generation $\mathrm{Z}$ (Median $=21-30 \mathrm{~h}$ per week) compared to the other generations (Median $=11-20 \mathrm{~h}$ per week).

Additionally, analyses of self-reporting based on the Money spent on video games per month resulted in a decreasing trend of reporting with age, displaying the lowest amount for Baby Boomers (Median $=11-30 \$$ ) in comparison with Generation Z (Median=31-60 \$).

Kendall's tau for non-parametric correlations with age and generation index (categorical number associated with each generation) were particularly high. Table 2 presents Kendall's tau values of Age and Generation index correlates with measured variables. Among those, strongest significant correlation was observed between generation index and 'prelvl30 playtime hrs' at $\tau=0.326$, which is significant at the 0.01 level (2-tailed). 
Correlations with generation index were also significant (2-tailed at the 0.01 level) for the number of days played ('daysplayed') and the amount of time (hrs) played ('playtime hrs') (both $\tau=0.324)$, as well as 'days in group' $(\tau=0.209)$, while 'max gear score' $(\tau=0.177)$, 'playtime group hrs' $(\tau=0.153)$, 'prelvl30 playtime group hrs' $(\tau=0.139)$, 'max level' $(\tau=0.121)$, self-reported hours per week $(\tau=-0.084)$, NPC Closeness $(\tau=0.057)$, Agency $(\tau=0.055)$, and Mastery $(\tau=-0.050)$.

Age showed similar power in correlations with 'daysplayed' $(\tau=0.317)$ 'playtime hrs' $(\tau=0.314)$, 'daysingroup' $(\tau=0.205)$, 'max gear score' $(\tau=0.171)$, 'playtime group hrs' $(\tau=0.149)$, 'prelvl30 playtime group hrs' $(\tau=0.138)$, 'max level' $(\tau=0.115)$, self-reported hours per week $(\tau=-0.079)$, NPCCloseness ( $\tau=0.060)$, Agency $(\tau=0.053)$, and Mastery $(\tau=-0.049)$.

\subsection{Correlations for Age and Generation Index}

We employed a series of machine learning techniques to further examine the significance of behavioral and self-reported measures in prediction of Age and generational index. As this testing conditions include 64 classes of age in 4 classes of generations, a random attempt at predicting age and generation will have baseline accuracies of $1.56 \%$ and $25 \%$ respectively.

First, we used a step forward neural network, which is a logistic regression classifier where the input is first transformed using a learnt non-linear transformation. This transformation projects the input data into a space where it becomes linearly separable. This intermediate layer is referred to as a hidden layer (Rumelhart et al. 1986).

In this experiment, a multilayer perceptron with 13 neurons in the hidden layer yielded a $30.00 \%$ accurate model in predicting age based on the input variables, including 'playtime hrs' (Predictor Importance $=0.11$ ), Competence $(\mathrm{PI}=0.10)$, 'prelvl30 playtime hrs' $(\mathrm{PI}=0.08)$, Relatedness $(\mathrm{PI}=0.07)$, 'playtimegroup hrs' $(P I=0.06)$, Growth and 'daysplayed' (both $\mathrm{PI}=0.05)$, and Closeness $(\mathrm{PI}=0.04)$. For the prediction of generation, a hidden layer consisting of 5 neurons reached the accuracy of $24.10 \%$ with the variables of 'playtime hrs' $(\mathrm{PI}=0.13)$, 'prelv130 playtime hrs' $(\mathrm{PI}=0.09)$, 'daysplayed' and 'max level' (both $\mathrm{PI}=0.07)$, as well as 'prelvl30 playtime group hrs' $(\mathrm{PI}=0.05)$.

The support vector machine regression module, which aims to fit the best regression line within a margin of error, was fed with all aforementioned input variables, predicted the age of our participants with a $26.05 \%$ success rate, compared to prediction of generation, which was accurate at $58.31 \%$ level. This difference in accuracy gains might be due to the number of categories for prediction (4 classes of generation and 64 classes of age in this study).

The linear model with a forward stepwise selection produced a $25.40 \%$ accurate model in predicting age, with predictors such as 'playtime hrs' $(\mathrm{PI}=0.28)$, 'playtime group hrs' $(\mathrm{PI}=0.27)$, Agency and 'daysplayed' (both PI =0.08), self-reported hours per week $(\mathrm{PI}=0.07)$, Emotional Presence $(\mathrm{PI}=0.05)$, as well as Growth $(\mathrm{PI}=0.04)$. 
For generation, the accuracy was at a $30.70 \%$ range, with the predictors 'playtime hrs' $(\mathrm{PI}=0.28)$, self-reported hours per week $(\mathrm{PI}=0.11)$, 'playtime group hrs', Agency and 'daysplayed' (all PI $=0.10)$, Emotional Presence $(\mathrm{PI}=0.06)$, as well as Growth and Competence (both PI $=0.05$ ).

Finally, a multimodal ensemble, which uses a supervised learning algorithm that combines a set of classifiers into a meta-classifier by taking weighted voting of their prediction for the final forecast, for age and generation prediction respectively produced $55.30 \%$ and $58.70 \%$ accurate results which were the highest rates among all tested models. Please refer to "Appendix 2" for graphs showing model accuracy gain for prediction of age and generation.

\section{Discussion}

This paper has presented the results from a study focusing on two large scale surveys deployed to players of Tom Clancy's The Division 2 (Ubisoft 2019), an online multiplayer shooter played by millions of players of different ages, and provides insight into the various factors relating to four different generational cohorts: Baby Boomers, Generation X, Millennials and Generation Z.

In summary, older players feel more agentic in the game, less masterful, closer to non-playable characters, and feel more present in the narrative than the younger generations. Additionally, even if older players perceive the game to provide them with more meaningful and believable options, the feedback provided by the game does not make them feel competent and effective.

The results from this study have shown that Agency, Mastery and feeling close to fantasy elements of the game, are the most discriminating measures of need satisfaction scores among generations. As a Previous study (Salmon et al. 2017) has shown, older adults prefer games that are easier to learn, challenging and single player. Our results confirm this finding by reporting a positive correlation between age and players' perceived agency and closeness to non-playable characters and narrative presence in the game. On the other hand, the reported negative correlation of age with the perceived Mastery suggests that the older players consider themselves less competent in the game because of either an agerelated decay in game performance or one's perception of it. Future studies could examine the relationship between game performance metrics and age. We also showed that duration of play and playing in groups correlates positively with age. Playtime is among the most popular measures of engagement (Wirth et al. 2013) as well as player churn prediction (Bertens et al. 2017). More playtime for older adults other than more available free time for the retired individuals, could be connected to the trend of lower perception of Mastery among older generations. Older generations may be compensating for their lower perception of Mastery by spending more time in the game, specifically when they reportedly feel more agentic and present in the game narrative. 
Connecting measures of Need satisfaction (UPEQ), playtime behavior and self-reported gaming habits of the players of different age groups, gives this study a unique perspective into generational differences in expectation, approach and perception that varies as video game audiences mature. This leads to various suggestions for the industry, academics and policy makers.

For game developers, this study argues that playtime and number of days played are not necessarily due to higher engagement, but perhaps the generational index of that population (see Sect. 4.1). Although, future research should consider further investigation relating to higher playtimes of both the Baby Boomers and Generation $\mathrm{X}$ cohort and their respective potential obsession with this medium as discussed by Przybylski et al. (2009).

For game designers, the results show that there are significant differences in perceived need satisfaction among generations, suggesting that certain generations may assess the game differently (which is not reflected in the single measure of rating) or have different aptitudes for subfactors of UPEQ. Future studies may explore multiple games between and within subjects.

For researchers and policy makers, the current study has established that age and generational index is indeed a factor of importance in the study and rapport of online gaming populations and their corresponding perception and behavior, and further investigation into socio-political factors related to this phenomenon is therefore advised.

We will now discuss Play time (behavioral) and UPEQ (need satisfaction) separately in the proceeding sections to outline the implications of our results.

\subsection{Play Time}

Among the behavioral measures, the majority of the cross-generational pairwise comparisons were significant (amounts to $78 \%$ of the comparisons), with predominantly Baby Boomers having the highest and Generation $\mathrm{Z}$ the lowest averages, and other generations following the trend. The positive correlation of age with most of the behavioral measures confirms this claim.

The results described in the results section, such as significant differences in playtime means across generation, correlations between measures and predictor importance of them when using machine learning techniques, suggest that both age and generation index are in fact indicators of the amount of time players spent in TD2. Playtime is considered to be among the most important factors in the game analytics and player churn prediction (Sifa et al. 2014). The 'Playtime' data from this study highly correlates with, and is the most important predictive factor of, age and generation in multiple methods.

Although the power of this impact is not overwhelming (correlation coefficient for generation and 'prelvl30 playtime hrs' is $\tau=0.326$; with 'playtime hrs' and 'days played' is both $\tau=0.324$ ), it pertains to three distinct aspects of time spent in the 
game. The data analysis showed that the more mature audience generally engage with the game more frequently, spend more time in the game, take more time to complete the game (playtime per level) and stay in the game for additional content, consistent with findings of Salmon et al. (2017).

Frequency of engagement, which was measured by counting the number of days in which the player started at least one game session, is an important factor for game developers since it impacts server populations, loading and login times on the game side (Suznjevic et al. 2014). Furthermore, the more frequent interaction a player has with the game can also help the player to remember the often-complex control scheme and economic logic of the game (Jakesch and Carbon 2012). Spending more time in the game is a major goal pursued by the industry (Canossa et al. 2019) and sometimes deemed as a potential for obsessive attachment (Przybylski et al. 2009), which in turn has also been connected to a better sense of achievement and success among boys (Hamlen 2010). Moreover, although previous studies have shown that playtime is related to rating of the game and player engagement (Azadvar and Canossa 2018; Wirth et al. 2013) in this study, rating was not a factor of age, generation or playtime. The only significant difference in averages for rating was that Baby Boomers' rating of the game was higher than Millennials' by $2.75 \%$.

While the Baby Boomers, on average, logged into the game 67 more days than the average Generation $\mathrm{Z}$ player (35 days), Baby Boomers also stayed in the game on average for $50+$ min more per day, playing more than the average Generation $\mathrm{Z}$ player. The higher game engagement amounts to an average of $400 \mathrm{~h}$ spent in the game by Baby Boomers compared to $100 \mathrm{~h}$ for Generation $\mathrm{Z}$ and $200 \mathrm{~h}$ overall average.

Previous research has shown that older adult gamers not only play longer, but also pay more for games and are more willing to pay (Schultheiss 2012). Results of the current study confirm that Baby Boomers play significantly more and share with Generation $\mathrm{X}$ the highest frequency in purchasing additional content, though in selfreported measures they reported the most modest amount of money spent in a month on video games compared to the other generations (Millennials reported the highest amount spent). We suggested that this trend may be a sign or a consequence of more income and available leisure time for the aging population, according to the existing literature (Pearce 2008; Marston 2012).

\subsection{Need Satisfaction (UPEQ)}

In the perceived need satisfaction (UPEQ scores), there were significant differences in $33 \%$ of the comparisons between the 4 generations. On these measures, Baby Boomers tend to score the highest, while Millennials score the lowest. Baby Boomers felt significantly closer to the non-playable characters and felt more present in the game narrative than other generations, supporting a previous study (Pearce 2008) suggesting that Baby Boomers prefer games with a story. 
Given the measures of Autonomy, according to KW H-tests, scores on Playstyle and Agency differed significantly between Millennials (lowest scores) and other generations, with Baby Boomers standing out with the highest score. This might be related to Baby Boomers' higher scores for fantasy elements of the game (NPC Closeness and Narrative Presence) due to the high correlation with the sense of autonomy (agency and playstyle). This relationship implies that by investing more in the fantasy one may perceive game options to be more meaningful (Deci and Ryan 2012).

For Mastery (including items like "I felt competent at the game."), Generation $\mathrm{X}$ scores were significantly lower than those of the other generations. This finding emphasizes that Generation $\mathrm{X}$ players perceive themselves to be less competent though the measure of game performance (max_gearscore) was significantly higher for them compared to Millennials and Generation Z players. The mismatch observed in this case could be a result of designing games with the younger audience as a target and therefore missing nuances of how older adults interact with this medium (De Schutter and Vanden Abeele 2010).

Another point worthy of discussion is how Baby Boomers have both the highest overall playtime and group playtime; however, the ratio, which gives 'group playtime percent' is the lowest for them (at 34.92\%) and is the highest for Generation Z players $(48.31 \%)$. The same argument is valid when we look at pre-level 30 group playtime percent. In other words, the percentage of social play is decreasing with age. However, there were no significant differences in UPEQ's Relatedness score (perception of social aspects of the game) among generations. As we have shown, higher scores of narrative presence and NPC Closeness may be a signal that the older adults prefer story-based games which are usually played individually. Another explanation might be the lack of technological familiarity (IJsselsteijn et al. 2007) or reluctance to engage with online communications due to safety risks (Agosto and Abbas 2017). Future studies may explore this emerging pattern to examine its generalizability to other games and what it implies for game development targeting older adults.

\subsection{Limitations}

The limitations of this study include different forms of respondents' selection for our surveying tool. Participants had to sign up for Ubisoft email communications and fulfill a playtime requirement and completion of the survey to be included in our study. Participants who chose to respond to the survey specified their age and other self-reported data without any form of validation.

This study only included respondents from one commercial video game, Tom Clancy's The Division 2, 4 months after its release. The nature of the game, the type of audience it attracts and the time frame for data collection may have skewed our dataset and emphasizes the need for further investigation of a more diverse products, samples and time frames. 
The behavioral measures could consist of a more granular set, in order to connect closer to need satisfaction types and to build better predictors. Current variables largely revolve around playtime and progression, while they could include performance metrics, activity types and sequence of player actions, something that could potentially reinforce player modeling attempts.

This study took an exploratory approach to available data sets, future studies may test the claims of this article as hypothesis in more controlled testing environments.

\section{Conclusions}

The current study employs a self-determination based model (UPEQ) to study a large number of players of a commercial online video game, across multiple generations. Multimodal data (self-reported habits, need satisfaction according to UPEQ, and in-game behavior) was used to increase reliability of the tested concepts.

Although rarely attempted, Marston and colleagues studied engagement among the aging population for an exercise game (Marston et al. 2016). However, we expanded on that premise by evaluating different aspects of player need satisfaction in a larger scale and by adding data from other generations for meaningful comparisons. The results present a significant difference in the mean values of the behavioral and need satisfaction measures. Significant correlations and predictive importance of these measures also facilitated our exploration in the effects of age and generation on play behavior and perception of need satisfaction.

Our data showed a trend that significantly higher playtime, days played and group playtime was correlated positively and significantly more for older adults. We conclude that older generations take more time going through the content and that they play more on average although their tendency to play with others decreases with age according to the presented data.

Intergenerational comparison of perceived need satisfaction also found that older gamers feel more agentic, present in the narrative, closer to non-playable characters but feel less competent at the game. These findings may imply an aptitude for a more narrative driven experience for older players who also prefer to take more time to play the same amount of content compared to younger audiences.

Significant differences between generations when evaluating the game in regard with Need Satisfaction, also implies that single measures of player engagement (e.g. Ratings or Playtime) are not able to capture the depth of experience and psychological need satisfaction of multiple generations playing a commercial video game. While this study provides insight into generational differences of players of a single commercial video game, investigation of between-and-within subjects as well as between-and-within video games could shed more light on the nature and causality of the explored correlations.

Acknowledgements Open access funding provided by Malmö University. This study is partially funded via Malmo University DDS research school by the Knowledge Foundation (KKS) and by Ubisoft Entertainment Sweden. 
Open Access This article is licensed under a Creative Commons Attribution 4.0 International License, which permits use, sharing, adaptation, distribution and reproduction in any medium or format, as long as you give appropriate credit to the original author(s) and the source, provide a link to the Creative Commons licence, and indicate if changes were made. The images or other third party material in this article are included in the article's Creative Commons licence, unless indicated otherwise in a credit line to the material. If material is not included in the article's Creative Commons licence and your intended use is not permitted by statutory regulation or exceeds the permitted use, you will need to obtain permission directly from the copyright holder. To view a copy of this licence, visit http://creativecommons.org/licen ses/by/4.0/.

\section{Appendix 1: UPEQ}

\section{UPEQ main}

Playstyle

Agency

Growth

Mastery

Closeness

Interdependence

NPC closeness

\section{UPEQ extention}

Narrative

Emotional presence

Transportation
I was free to decide how I wanted to [play]

I could approach [the game] in my own way

The game allowed me to [play] the way I wanted to

I had important decisions to make when [playing]

The choices I made while [playing] influenced what happened

My actions had an impact on the [game]

With time, I became better at [playing]

My [gaming] abilities have improved since the beginning

My mastery of the [game] improved with practice

I was good at [playing]

I felt competent at [playing]

I felt very capable and effective when [playing]

I really like the people I play with

I consider players I regularly interact with to be my friends

Other players are friendly towards me

What other players did in the game had an impact on my actions

I had to adapt my actions to other players' actions

I was paying attention to other players' actions

I felt close to some of the characters

I was bonding with some of the characters

I cared about what happens to some of the characters

My characters' motivations were coherent with my actions

My actions are coherent with my characters'

Everything I did as the main character felt right

I am impacted emotionally by events in the game

When I accomplished something in the game I experienced genuine emotions I experience feelings as deeply in the game as I have in real life

When playing the game, I feel transported to another time and place

Exploring the game world feels like taking an actual trip to a new place

When moving through the game world I feel as if I am actually there 


\section{Appendix 2: Gain Graphs}
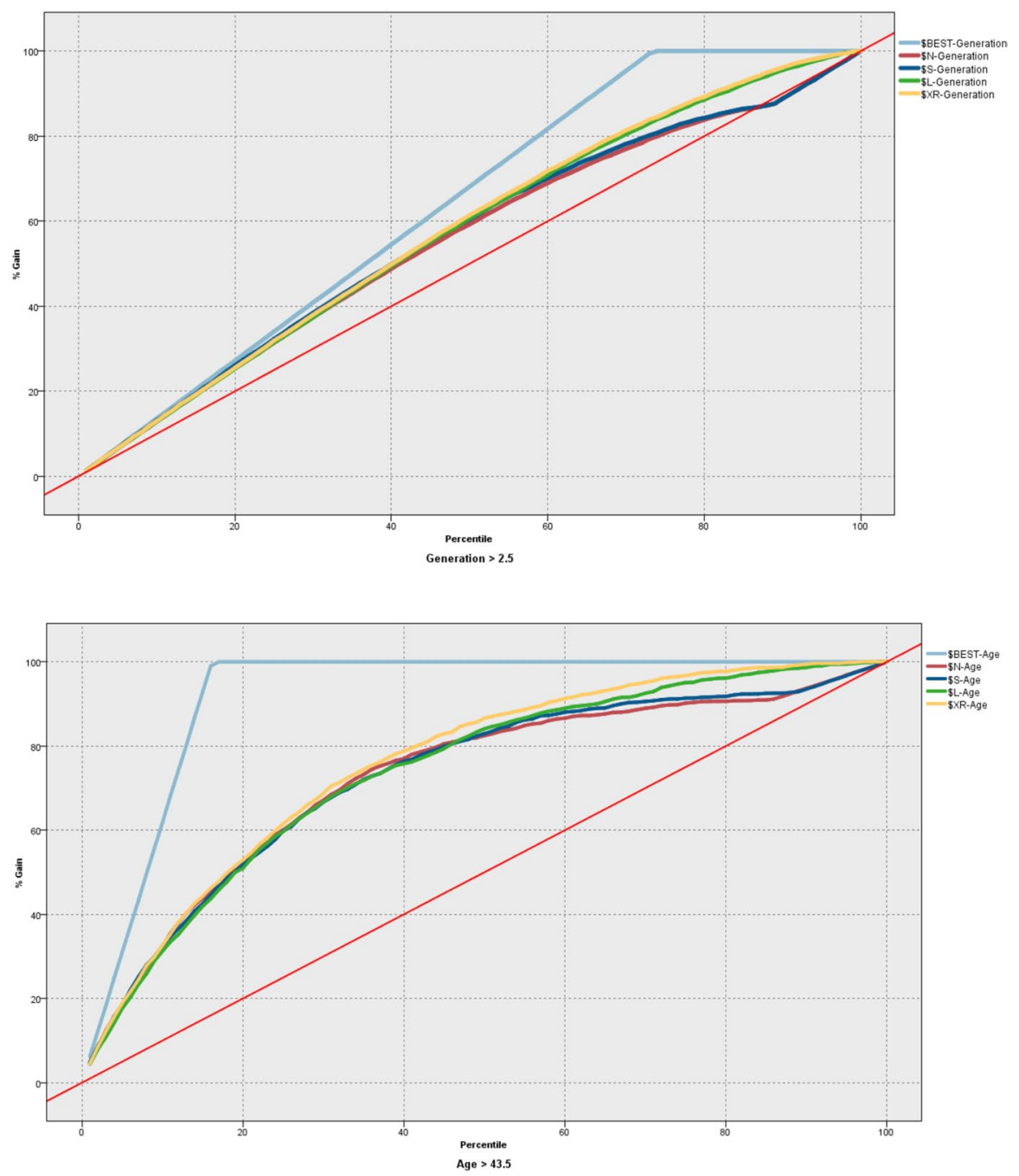


\section{References}

Agosto, D. E., \& Abbas, J. (2017). "Don't be dumb-That's the rule I try to live by": A closer look at older teens' online privacy and safety attitudes. New Media \& Society, 19(3), 347-365.

Azadvar, A., \& Canossa, A. (2018). UPEQ: Ubisoft perceived experience questionnaire: a self-determination evaluation tool for video games. In Proceedings of the 13th international conference on the foundations of digital games (p. 5). ACM.

Bertens, P., Guitart, A., \& Periáñez, Á. (2017). Games and big data: A scalable multi-dimensional churn prediction model. In 2017 IEEE conference on computational intelligence and games (CIG) (pp. 33-36). IEEE.

Canossa, A., Azadvar, A., Harteveld, C., Drachen, A., \& Deterding, S. (2019). Influencers in multiplayer online shooters: Evidence of social contagion in playtime and social play. In Proceedings of the 2019 CHI conference on human factors in computing systems (p. 259). ACM.

Canossa, A., Badler, J. B., El-Nasr, M. S., Tignor, S., \& Colvin, R. C. (2015). In your face (t) impact of personality and context on gameplay behavior. In FDG.

Carnagey, N. L., Anderson, C. A., \& Bushman, B. J. (2007). The effect of video game violence on physiological desensitization to real-life violence. Journal of Experimental Social Psychology, 43(3), 489-496.

Cortes, C., \& Vapnik, V. (1995). Support-vector networks. Machine Learning, 20(3), 273-297.

Deci, E. L., \& Ryan, R. M. (2012). Self-determination theory. In P. A. M. Van Lange, A. W. Kruglanski, \& E. T. Higgins (Eds.), Handbook of theories of social psychology (pp. 416-436). Sage Publications Ltd. https://doi.org/10.4135/9781446249215.n21.

De la Hera, T., Loos, E., Simons, M., \& Blom, J. (2017). Benefits and factors influencing the design of intergenerational digital games: A systematic literature review. Societies, 7(3), 18.

De Schutter, B. (2011). Never too old to play: The appeal of digital games to an older audience. Games and Culture, 6(2), 155-170.

De Schutter, B., \& Vanden Abeele, V. (2010). Designing meaningful play within the psycho-social context of older adults. In Proceedings of the 3rd international conference on fun and games (pp. 84-93).

Dietterich, T. G. (2000). Ensemble methods in machine learning. In International workshop on multiple classifier systems (pp. 1-15). Springer, Berlin.

Dill, K. E., \& Dill, J. C. (1998). Video game violence: A review of the empirical literature. Aggression and Violent Behavior, 3(4), 407-428.

Entertainment Software Association. (2018). Sales, demographic and usage data. Essential facts about the computer and video game industry. Washington, DC: Entertainment Software Association.

Fisher, S. (1994). Identifying video game addiction in children and adolescents. Addictive Behaviors, 19(5), 545-553.

Green, C. S., \& Bavelier, D. (2003). Action video game modifies visual selective attention. Nature, 423(6939), 534.

Hamlen, K. R. (2010). Re-examining gender differences in video game play: Time spent and feelings of success. Journal of Educational Computing Research, 43(3), 293-308.

Han, D. H., Hwang, J. W., \& Renshaw, P. F. (2011). Bupropion sustained release treatment decreases craving for video games and cue-induced brain activity in patients with internet video game addiction. Psychology of Popular Media Culture, 1(S), 108-117. https://doi.org/10.1037/2160-4134.1.S.108.

Hong, S. H. (2015). When life mattered: The politics of the real in video games' reappropriation of history, myth, and ritual. Games and Culture, 10(1), 35-56.

Huang, J., Yan, E., Cheung, G., Nagappan, N., \& Zimmermann, T. (2017). Master maker: Understanding gaming skill through practice and habit from gameplay behavior. Topics in Cognitive Science, 9(2), 437-466.

IJsselsteijn, W., Nap, H. H., de Kort, Y., \& Poels, K. (2007). Digital game design for elderly users. In Proceedings of the 2007 conference on future play (pp. 17-22).

Ivory, J. D., \& Kalyanaraman, S. (2007). The effects of technological advancement and violent content in video games on players' feelings of presence, involvement, physiological arousal, and aggression. Journal of Communication, 57(3), 532-555.

Jakesch, M., \& Carbon, C. C. (2012). The mere exposure effect in the domain of haptics. PLoS ONE, 7(2), 31215. 
Jenson, J., \& de Castell S. (2018). Videogames and learning: Ethics, ontology and epistemology. In International handbook of philosophy of education (pp. 1321-1335). Springer, Cham.

Kendall, M. G. (1938). A new measure of rank correlation. Biometrika, 30(1/2), 81-93.

Kent, S. L. (2010). The ultimate history of video games: From Pong to Pokemon and beyond the story behind the craze that touched our lives and changed the world (Vol. 2). New York: Three Risvers Press.

Kruskal, W. H., \& Wallis, W. A. (1952). Use of ranks in one-criterion variance analysis. Journal of the American Statistical Association, 47(260), 583-621.

Marston, H. R. (2012). Older adults as 21st century game designers. The Computer Games Journal, 1 , 90. https://doi.org/10.1007/BF03392330.

Marston, H., \& Graner-Ray, S. (2016). Older women on the game: Understanding Digital game perspectives from an ageing cohort. In E. Domínguez-Rué \& L. Nierling (Eds.), Ageing and technology (pp. 67-92). Bielefeld: Transcript Verlag. https://doi.org/10.14361/9783839429570-004.

Marston, H. R., Kroll, M., Fink, D., \& Gschwind, Y. J. (2016). Flow experience of older adults using the iStoppFalls exergames. Special Issue: Gaming \& Aging Games \& Culture Journal, 11(1-2), 201-222. https://doi.org/10.1177/1555412015605219.

Mäyrä, F. (2008). An introduction to game studies. Thousand Oaks: Sage.

Pearce, C. (2008). The truth about baby boomer gamers: A study of over-forty computer game players. Games and Culture, 3(2), 142-174.

PEW Center. (2010). Millennials: A portrait of generation next. Washington: PEW Center.

Prensky, M. (2003). Digital game-based learning. Computers in Entertainment (CIE), 1(1), 21.

Przybylski, A. K., Weinstein, N., Ryan, R. M., \& Rigby, C. S. (2009). Having to versus wanting to play: Background and consequences of harmonious versus obsessive engagement in video games. CyberPsychology \& Behavior, 12(5), 485-492.

Salmon, J. P., Dolan, S. M., Drake, R. S., Wilson, G. C., Klein, R. M., \& Eskes, G. A. (2017). A survey of video game preferences in adults: Building better games for older adults. Entertainment Computing, 21, 45-64.

Sifa, R., Bauckhage, C., \& Drachen, A. (2014). The playtime principle: Large-scale cross-games interest modeling. In 2014 IEEE conference on computational intelligence and games (pp. 1-8). IEEE.

Schotter, A., \& Sopher, B. (2003). Social learning and coordination conventions in intergenerational games: An experimental study. Journal of Political Economy, 111(3), 498-529.

Schultheiss, D. (2012). "Entertainment for retirement?": Silvergamers and the internet. Public Communication Review, 2(2), 62-71. https://doi.org/10.5130/pcr.v2i2.2778.

Sherry, J. L. (2001). The effects of violent video games on aggression: A meta-analysis. Human Communication Research, 27(3), 409-431.

Skoric, M. M., Teo, L. L. C., \& Neo, R. L. (2009). Children and video games: Addiction, engagement, and scholastic achievement. Cyberpsychology \& Behavior, 12(5), 567-572.

Suznjevic, M., Saldana, J., Matijasevic, M., Fernández-Navajas, J., \& Ruiz-Mas, J. (2014). Analyzing the effect of tcp and server population on massively multiplayer games. International Journal of Computer Games Technology, 2014, 1-17. https://doi.org/10.1155/2014/602403.

Ryan, R. M., Rigby, C. S., \& Przybylski, A. (2006). The motivational pull of video games: A self-determination theory approach. Motivation and Emotion, 30(4), 344-360.

Rumelhart, D. E., Smolensky, P., McClelland, J. L., \& Hinton, G. (1986). Sequential thought processes in PDP models. Parallel Distributed Processing: Explorations in the Microstructures of Cognition, 2, 3-57.

Ubisoft. (2019). Tom Clancy's The Division 2, computer program. Ubisoft Entertainment Sweden: Malmö.

Wirth, W., Ryffel, F., Von Pape, T., \& Karnowski, V. (2013). The development of video game enjoyment in a role playing game. Cyberpsychology, Behavior, and Social Networking, 16(4), 260-264.

Zerlang, J. (2017). GDPR: A milestone in convergence for cyber-security and compliance. Network Security, $2017(6), 8-11$. 